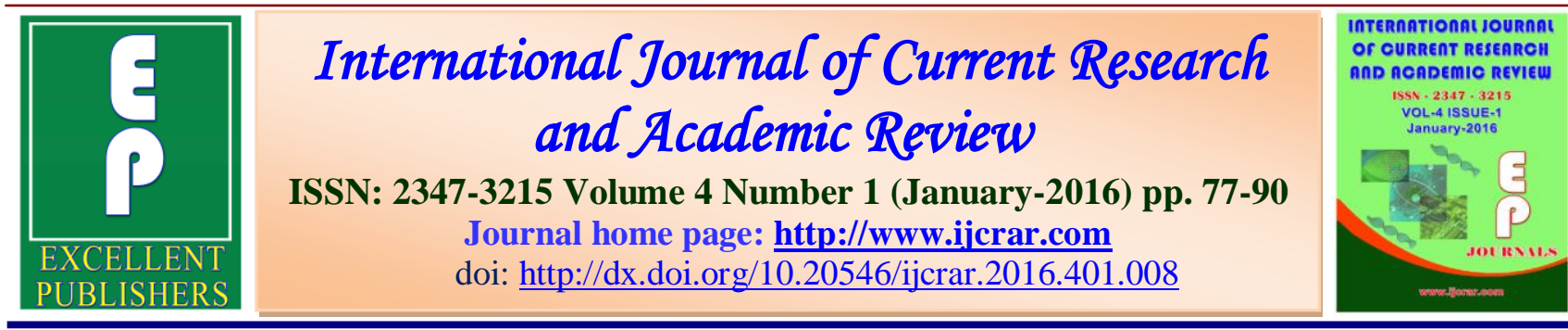

\title{
Studying the Interaction between the CME Magnetic Field and Jupiter's Magnetic Field by Simulation
}

\author{
Wafaa A.Zaki* and Zainab A. Ali \\ University of Kirkuk, College of Science, Department of Physics, Iraq \\ *Corresponding author
}

KEYWORDS

Magneto-

hydrodynamic

model: MHD,

Numerical simulation,

Coronal mass

ejections: CME;

Magnetosphere of

Jupiter

\section{A B S T R A C T}

In this investigation a simulation of coronal mass ejections CMEs from the corona of the Sun until it reaches Jupiter has been presented, by taking CME of kind halo $\left(360^{\circ}\right)$ with high speed which are in the form of events that expected to be interacting with the magnetic field of Jupiter and this happen in the two steps; First, simulation by using the ideal magneto hydrodynamic MHD model for the CMEs itself from the beginning of their growth and spread in the interplanetary from the Sun until are reaches Jupiter; Second, simulation of the interaction between the magnetic field of Jupiter and CME has been achieved by using semi-relativistic MHD. All these approaches were held for the rising phase of solar cycle 24 specifically for the years 2011 and 2014. All data were derived from Large Angle and spectrometric Coronagraph Experiment (LASCO), which is one of the scientific systems in the spacecraft Solar and Heliospheric Observatory ( $\mathrm{SOHO}$ ) that was located close lagrangain point L1 where the data related to Jupiter were derived from Voyager. The numerical model used in the present simulation was based on Leap-Frog method which is an explicit differential equation using Matlab program and it had been found that the different time stepping methods are Optimal. It had been concluded that the acceleration of the energetic particle not only in the interplanetary, but also in the location of the event in the corona of the sun, and it had been found that the event increased gradually with adopted years (2011 and 2014). Also the computational MHD is a dynamically evolving field and going beyond ideal MHD Creates a new algorithm where the energy conservation will be violated by a nonconservation MHD model which is non by semi-relativistic MHD model.

\section{Introduction}

Solar activity presented as two mainly types; sun spots and solar wind. Solar wind is consisting of three main types flare, coronal mass ejections (CMEs) and solar 
energetic particles (SEP). The solar wind flows outward supersonically to great distances, filling a region known as the Heliosphere, an enormous bubble-like volume surrounded by the interstellar medium. The sun's corona, or extended outer layer, is a region of plasma that is heated to over a million degrees Celsius (1).

The total number of particles carried away from the sun by the solar wind is about $1.3 \times 10^{36}$ per second (2). Thus, the total mass loss each year is about $(2-3) \times 10^{-14}$ solar masses (3), or about one billion kilograms per second. This is equivalent to losing a mass equal to the Earth every 150 million years (4). However, only about $0.01 \%$ of the Sun's total mass has been lost through the solar wind (5). Other stars have much stronger stellar winds that result in significantly higher mass loss rates. During solar minimum, the observations clearly show that the solar wind exhibits two modes of outflow as shown below:

The fast wind, associated with polar coronal holes, with outflow speeds of $\approx 800 \mathrm{~km} \mathrm{~s}^{-1}$

The slow wind with outflow speeds of $\approx 400$ $\mathrm{km} \mathrm{s}^{-1}$ associated with equatorial regions (6).

The main goal of this study is to identify the solar events which represent a CME of type halo and follow its growth from sun until it reaches Jupiter and studying its magnetic fields by a numerical simulation using the magneto-hydrodynamic model (MHD) in two stages. First stage: The transformation of CME from sun toward Jupiter until it reaches the outer shell of the magnetosphere of Jupiter and this case is the conservative case, so in this interval the ideal MHD will be recommended for simulation. Second stage: After the arrival of CME to the outer shell of the magnetosphere of Jupiter so the reconnection process will exist and all plasma of the CME will confined within the magnetic field lines of the Jupiter magnetosphere (non-conservative), so in this process the semi-relativistic MHD will take place in this simulation. All the observations that have been used in this Study are for solar cycle 24 .

\section{Data Handling}

In this study 2 events has been selected from SOHO/ERNE (7). The selected events have a clear background with intensities $>10^{-3} \mathrm{~cm}^{-2} \mathrm{sr}^{-1} \mathrm{~s}^{-1} \mathrm{Mev}^{-1}$ for energy (1$116 \mathrm{MeV})$, detected by SOHO/ERNE from January 2011 till December 2014 during solar cycle 24 . The Information of intensitytime profile of SEPs comes from two ERNE detectors, High Energy Detector (HED) and Low Energy Detector (LED) (8). The identification of protons is based on an onboard algorithm, which provides intensities in the energy ranges $1.3-14 \mathrm{MeV}$ for LED and 13- $140 \mathrm{MeV}$ for HED, within one minute time resolution. The particle data is accessible through the ERNE Data finder (7). The widest possible energy range starting from $1 \mathrm{MeV}$ up to $116 \mathrm{MeV}$ of each event are used to see the differences in the intensity-time profile in each channel and whether they are the same for all events. The present choice of these events are according to photometric observation on which were obtained from $\mathrm{SOHO}$, so the calculations are illustrated as in table (1) which shows the SEPs onset and the associated phenomena time for all adopted events. The onset time for the first peak in the SEP events must be found and then the release time for the first non-scattered proton traveling on the nominal path length of 1.2 AU for all the events has been calculated. All the observed CMEs are listed in Table (1) and (2) are of the first appearance in the LASCO field of view, speed $(V$ in $\mathrm{km} / \mathrm{s})$, height $\left(R_{\Theta}\right)$. The 
speed and height were measured in the sky plane, so they are expected to be lower limits to the actual values. The speeds were obtained by a straight-line fit to the heighttime measurements and hence are average values within the LASCO field of view $\left(\sim 2-32 R_{s}\right)$. LASCO consists of three coronagraphs, designated $\mathrm{C} 1, \mathrm{C} 2$ and $\mathrm{C} 3$, with respective overlapping fields of view of 1.1 to $3,1.7$ to 6 , and 3.7 to 32 solar radii, here $R_{s}$ denotes the solar radius, or 696 million $\left(6.96 \times 10^{8}\right)$ meters. The mass and energy calculated from coronagraph images.

In the table (3) the shaded values indicate to the arrival times (in day) to the various distances in Astronomical Units and the speed of the coronal mass ejection (CME) indicated in the first column. The distance from Sun to the Jupiter ( 780 million $\mathrm{km}$ ) is defined to be exactly 5.2 Astronomical Unit (5.2 AU). A 'Halo' CME is an explosive expulsion of heated gas from the sun directed towards Jupiter.

The main results produced in this research are the physical properties of the coronal mass ejection which change due to the interaction with the Jupiter's magnetic field. This interaction is described by the main conservation laws (equations (1) to (5)). The instantaneous solution of this system of differential equations will result in the basic physical properties of the system which are presented in this study.

\section{Theoretical Approaches and Numerical Simulation}

The MHD model is the extension of fluid dynamics to electrically conducting fluids such as plasmas. And this model is very convenient for the numerical simulation of fluid such as the $\mathrm{CME}$ and the magnetic fields. The corresponding MHD equations describe the evolution of macroscopic quantities such as density, bulk velocity, magnetic field and pressure of plasma flows. Various forms of MHD models have been extensively used in space physics to describe many different kinds of plasma phenomena, such as magnetic reconnection and solar wind interaction with different celestial objects, its equations consist of the macroscopic transport equations and the magnetic induction equation. The transport equations can be obtained by multiplying the Boltzmann equation with an appropriate function of velocity and then integrating over the velocity space (8). The induction equation is a combination of Maxwell's equations and the generalized Ohm's law. According to different assumptions made in the derivation, MHD models have various forms. In this study will rely on two models of the MHD model and the semi-relativistic MHD model. In the MHD approach the plasma is described by a set of fluid equations that describe the conservation of mass, energy and the evolution of the magnetic field (9).In ideal MHD, plasma is frozen into the magnetic field $=>$ field line topology conservation and magnetic reconnection is not possible (10), so in the electron adopted model, it had been assumed that the systems are composed of magnetized plasmas that behave as an ideal gas with a poly-tropic index, $\gamma=5 / 3$.The plasma is assumed to have infinite electrical conductivity so that the magnetic field is "frozen" into the plasma. Gravitational forces on the plasma are included, but only those forces due to the Sun; there is no selfgravitation of the plasma (11).

The state of plasma with mass density $(\rho)$, number of particle $(n)$, momentum $(q)$, pressure $(p)$, velocity $(v)$, and magnetic field $(B)$ at space $\boldsymbol{x}$ and time $\boldsymbol{t}$ is determined by the conservation law of ideal MHD. The equations of the ideal MHD describe the 
dynamics of plasma under the influence of a magnetic field, and are given by (12):

$$
\frac{\partial \rho}{\partial t}+\nabla \cdot(\rho v)=0
$$

$$
\frac{\partial u}{\partial t}-u \nabla u+\frac{1}{\rho} \nabla P+\frac{B}{4 \pi \rho} \nabla B=0
$$

$\frac{\partial B}{\partial t}=[\nabla \times[v \times B]]$

$\frac{\partial P}{\partial t}+v \frac{\partial \rho}{\partial x}+\gamma \rho \frac{\partial v}{\partial x}=0$

$e=\frac{\rho u^{2}}{2}+\frac{p}{\gamma-1}+\frac{B^{2}}{8 \pi}$

Semi-relativistic MHD is used to model Jupiter and Saturn where classical Alfven speed can be larger than c (Alfven speed can be huge).To obtain the semi-relativistic form, our primitive-variable equation set of the semi-relativistic MHD with anisotropic ion pressure and isotropic electron pressure is composed of many equations (13).

An alternative derivation leading to the semi relativistic MHD equations starts with the non-conservative form of the nonrelativistic MHD equations but with the electric force in the momentum equation and the displacement current in Ampere's law being kept. A form of this derivation was discussed by Boris (14). In this case the governing equations are:

$\frac{\partial \rho}{\partial t}+(u . \nabla) \rho+\rho(\nabla . u)=0$

$\frac{\partial B}{\partial t}+\nabla \times[-(u \times B)]=0$

$\frac{\partial p_{e}}{\partial t}+(u . \nabla) p_{e}+\frac{5}{3} p_{e}(\nabla \cdot u)=0 \ldots$

$$
\begin{aligned}
& \frac{\partial\left[\rho u+E \times B /\left(\mu_{0} c^{2}\right)\right]}{\partial t}+\nabla \cdot\left[\rho u u+\left(p_{\perp}+p_{e}\right) I+\right. \\
& \left.\left(p_{\|}-p_{\perp}\right) b b+\frac{B^{2} I}{2 \mu_{0}}+\frac{E^{2} I}{2 \mu_{0} c^{2}}-\frac{B B}{\mu_{0}}-\frac{E E}{\mu_{0} c^{2}}\right]=0
\end{aligned}
$$

And the energy equation is written as:

$$
\begin{aligned}
& \frac{\partial\left[e+E^{2} /\left(2 \mu_{0} c^{2}\right)\right]}{\partial t}+\nabla \cdot\left[u \left(e+p_{\perp}+p_{e}-\right.\right. \\
& \left.\left.\frac{B^{2}}{2 \mu_{0}}\right)+\left(p_{\|}-p_{\perp}\right) u \cdot b b+\frac{E \times B}{\mu_{0}}\right]=0
\end{aligned}
$$

The numerical model used in the present simulation was based on Leap-Frog method, which is an explicit method of good accuracy used for solving differential equations as mentioned here. Using Matlab to perform the present calculations made it easier to have control over I/O parameters as well as its good ability in dealing with large matrices. The main program calls many subroutines which perform various tasks such as applying initial and boundary conditions, solving the set of differential equations for the MHD system and plotting the results. The Leapfrog method is widely used to solve numerical problem with initialboundary values. It is attractive because it is simple with accuracy of second order, but most of all it has very good stability when computing oscillatory solutions (15).

This method is $2^{\text {nd }}$ order accurate in space and time, i.e. with accuracy $\left((\Delta t)^{2},(\Delta x)^{2}\right)$.

Two major steps of Leapfrog method explicit were performed in this study:

Using centered scheme on time and space derivative, such as,

$\frac{\partial \rho}{\partial t}=\frac{\rho_{i}^{n+1}-\rho_{i}^{n-1}}{\partial t} \quad, \quad \frac{\partial \rho}{\partial x}=\frac{\rho_{i+1}^{n}-\rho_{i-1}^{n}}{2 \Delta x}$ 
Where $n$ being the order of time step and $i$ is the step on spatial mesh grid.

Using the stability condition for space derivatives which is made by adding the expression of the time derivative for the current $n^{\text {th }}$ time state and $i^{\text {th }}$ space state. That is achieved by substituting the average of the variable state for the given degree of freedom, i.e., displacing the term $\rho_{i}^{n}$ in equation eq.(1) for one dimension by:

$\frac{\rho_{i+1}^{n}+\rho_{i-1}^{n}}{2}$,

Or $\rho_{i, j}^{n}$ for two dimension by:

$\frac{\rho_{i+1, j}^{n}+\rho_{i-1, j}^{n}+\rho_{i, j+1}^{n}+\rho_{i, j-1}^{n}}{4}$,

Or $\rho_{i, j, k}^{n}$ for three dimension by:

$\frac{\rho_{i+1, j, k}^{n}+\rho_{i-1, j, k}^{n}+\rho_{i, j+1, k}^{n}+\rho_{i, j-1, k}^{n}+\rho_{i, j, k+1}^{n}+\rho_{i, j, k-1}^{n}}{6}$.

\section{Applications and Discussion}

The initiation of a CME was simulated by local pressure and density enhancements in this study. This was primarily for the sake of numerical convenience and not because it is thought that pressure enhancement is the most likely mechanism for triggering the onset of CMEs. The goal of these first studies was more to test the ability of the MHD model to simulate solar wind disturbances than to better understand CMEs. The appropriate boundary conditions at the inner boundary has been specified (the spherical surface $r=R_{\Theta}$ ). The code solves a boundary-condition problem; hence a set of initial and boundary conditions are required. This set was chosen in order to have convergent solutions with physical meanings. The set of initial conditions were applied at the first step of the program and it is required to set-up the various matrices used during the calculations. The boundary conditions, on the other hand, were repeatedly applied during each loop of the code so that the limits of the calculated physical properties are always under control, thus they do not affect the present main results regarding to the initiation and propagation of the CME. Initial and boundary conditions used in the present work are listed in Table (5) and (6).

The results of physical properties of coronal mass ejection (1) are shown in Figures (1), (2) and (7). From equation (1) has been used in order to calculate the mass density of coronal mass ejection and it found that its value increasing whenever grow. From figure (3), it has been found that the peak was $\simeq 13 \times 10^{33}$ at $\mathrm{Z}=5 R_{\Theta}$ and become $\simeq$ $21 \times 10^{34}$ at $\mathrm{Z}=30 R_{\odot}$ where $\mathrm{z}$ represents the distance from the solar radius.

A net-velocity of a CME will be found which behave as shown in figure (2). The velocity at time (20:00) to start from the sun and the distance traveled by the time of arrival after (3.2 day) starting of the sun.

Equation (1) has been used in order to calculate the mass density of CME2 and it has been found that its value increasing whenever grown. From figure (3), it has been found that the peak was $\simeq 8 \times 10^{33}$ at $\mathrm{Z}=5 R_{\odot}$ and become $\simeq 16 \times 10^{34}$ at $\mathrm{Z}=30 R_{\odot}$ where $\mathrm{z}$ represent the distance from Sun in solar radius.

A net-velocity of coronal mass ejection will be found which behave as shown in figure (4). The velocity at time $(13: 25)$ to start from the sun and the distance traveled by the time of arrival after (7 day) Starting of the sun. 
When the coronal mass ejection (1 and 2) is near to the sun, it will be exposure to the electric and magnetic fields, where the coronal mass ejection is affected by two forces the electric force $F_{e}=q E$ and magnetic force $F_{B}=q v \times B$, the resultant of these forces is called Lorentz force, as shown in this equation: $F=q(E+v \times B)$

Where the Lorentz force $(F)$ in unit of $(\mathrm{N})$, the electric charge of particle (q) in unit of(columb), the electric field (E) in unit of (volt $/ \mathrm{m})$, linear velocity of particle (v) in unit of $(\mathrm{m} / \mathrm{sec})$ and The magnetic field (B) in unit of $(\mathrm{nT})$. From the previous equation, it was noted that the Lorentz force is proportional to the magnetic field. So the magnetic field increases as shown in figure (5).The net of force depends on the magnitude and direction of both velocity and magnetic field. Therefore, when B increases, it is expected that, at constant velocity and direction, the force will also increase leading to more conferment of the plasma of the sun.

Jupiter provides us with a relatively handy example of a rapidly rotating magnetospheric obstacle that is very different than that at Earth. The models were based on a combination of this large data base with results from magneto hydrodynamic (MHD) simulations of the effects of different coronal mass ejection (CMEs) dynamic pressures on the bow shock and magnetopause shapes and locations. It is briefly reviewed the simulation model and discussed the runs used to support this study.

The goal is to quantify the planetary magnetic field strength necessary to shield the planet from CME events; Jupiter's equatorial field is about $4.3 \mathrm{G}$. The CME does not penetrate more than $0.5 R_{p}$ above the surface as shown in Figure (7) and (8). This means that the planetary atmosphere is well-shielded from erosion by the magnetic field and dynamic pressure of the CME by even quite modest planetary magnetic fields of 0.5 and $1.0 \mathrm{G}$. When a CME is propagating through the solar corona, it expands and accelerates while pushing the ambient coronal plasma outward and interacting with its magnetic field. In our simulation, a similar scenario is obtained until the CME reaches the nose of the planetary magnetosphere. Then, the CME is deflected and adiabatically expands around the magnetosphere front and envelopes it from the sides (noticeable at $\mathrm{t}=01: 00 \mathrm{~h}$ ). The CME front is slowed down and the temperature at its front drops. Later on, at $\mathrm{t}$ $=01: 20 \mathrm{~h}$ the CME reaches the night side of the planetary magnetosphere, while detaching from part of the planetary magnetic tail. At this point, the size of the planetary magnetosphere, characterized by the low temperature bubble near the planet, is significantly decreased. Finally, at $\mathrm{t}=$ 03:00, the magnetosphere starts to recover from the initial impact by the $\mathrm{CME}$, it becomes larger, and its tail is stretched in the anti-stellar direction. This is due to the ambient plasma, which is now dominated by the radial propagation and expansion of the CME.

The magnetosphere is almost perpendicular in orientation to the direction of propagation of the CME, and the CME hits the magnetosphere from the side and not on its nose.This orientation involves a contact surface between the magnetosphere and the CME. When the magnetospheric orientation is changed from being perpendicular to the CME direction of propagation to being aligned with it, the magnetospheric nose at this stage is roughly at the sub-stellar point, with a steady bow shock in front of it. At Jupiter, the CME may not be much larger than the magnetosphere, but it can be considered as a plane wave. Initial and boundary conditions used in the present work are listed in Table (7) (16). 


\section{Int.J.Curr.Res.Aca.Rev.2016; 4(1): 77-90}

Table.1 Illustrate the Energy Channel, Onset Time, Release Time, and Height

\begin{tabular}{|c|c|c|c|c|c|}
\hline \multicolumn{2}{|c|}{ Events } & $\begin{array}{c}\text { Energy } \\
(\text { Mev })\end{array}$ & $\boldsymbol{t}_{\text {onset }}(\mathrm{UT})$ & $\begin{array}{c}\boldsymbol{t}_{\text {release }} \\
( \pm \mathbf{8})(\mathrm{UT})\end{array}$ & Height CME at C2 $\left(\boldsymbol{R}_{\odot}\right)$ \\
\cline { 1 - 2 } NO. & DD.MM.YY & & & & \\
\hline 1 & $(4-15) .03 .2011$ & 36.4 & $20: 55$ & $20: 27$ & 2.43 \\
\hline 2 & $(15-20) .04 .2014$ & 45.6 & $13: 35$ & $13: 10$ & 5.49 \\
\hline
\end{tabular}

Table.2 CME Linear Speed, Mass and Kinetic Energy

\begin{tabular}{|c|c|c|c|c|c|}
\hline NO. & \multicolumn{2}{|c|}{$\begin{array}{c}\text { First C2 Appearance } \\
\text { Date Time (UT) }\end{array}$} & $\begin{array}{c}\text { Linear Speed } \\
(\mathbf{K m} / \mathbf{s})\end{array}$ & $\begin{array}{c}\text { Mass } \\
(\mathbf{K g})\end{array}$ & $\begin{array}{c}\text { Kinetic Energy } \\
\text { (Joules) }\end{array}$ \\
\hline 1 & $2011 / 03 / 07$ & $20: 00: 05$ & 2125 & $1.7 \times 10^{13}$ & $3.9 \times 10^{25}$ \\
\hline 2 & $2014 / 04 / 18$ & $13: 25: 51$ & 1203 & $4.5 \times 10^{13}$ & $3.3 \times 10^{25}$ \\
\hline
\end{tabular}

Table.3 Estimating Halo Coronal Mass Ejection (CME) Arrival Time (t)

\begin{tabular}{|c|c|c|c|c|c|c|c|c|c|c|c|c|c|}
\hline Linea & \multicolumn{13}{|c|}{ Distance from the Sun in Astronomical Units } \\
\hline $\begin{array}{c}\text { Speed } \\
(\mathbf{K m} / \mathbf{s} \\
)\end{array}$ & & 0.5 & 1 & 1.5 & 2 & 2.5 & 3 & 3.5 & 4 & 4.5 & 5 & 5.2 & 5.5 \\
\hline 2125 & \multirow{2}{*}{$\begin{array}{l}t^{t} \\
\text { (day }\end{array}$} & $\begin{array}{c}0.408 \\
5\end{array}$ & $\begin{array}{c}0.817 \\
0\end{array}$ & $\begin{array}{c}1.225 \\
5\end{array}$ & $\begin{array}{c}1.634 \\
0\end{array}$ & $\begin{array}{c}2.042 \\
5\end{array}$ & $\begin{array}{c}2.451 \\
0\end{array}$ & $\begin{array}{c}2.859 \\
5\end{array}$ & $\begin{array}{c}3.268 \\
0\end{array}$ & $\begin{array}{c}3.676 \\
5\end{array}$ & $\begin{array}{c}4.085 \\
0\end{array}$ & $\begin{array}{c}4.248 \\
4\end{array}$ & $\begin{array}{c}4.493 \\
5\end{array}$ \\
\hline 1203 & & $\begin{array}{c}0.721 \\
6\end{array}$ & $\begin{array}{c}1.443 \\
2\end{array}$ & $\begin{array}{c}2.164 \\
7\end{array}$ & $\begin{array}{c}2.886 \\
3\end{array}$ & $\begin{array}{c}3.607 \\
9\end{array}$ & $\begin{array}{c}4.329 \\
5\end{array}$ & $\begin{array}{c}5.051 \\
0\end{array}$ & $\begin{array}{c}5.772 \\
6\end{array}$ & $\begin{array}{c}6.494 \\
2 \\
\end{array}$ & $\begin{array}{c}7.215 \\
8\end{array}$ & $\begin{array}{c}7.504 \\
4\end{array}$ & $\begin{array}{c}7.937 \\
3 \\
\end{array}$ \\
\hline
\end{tabular}


Int.J.Curr.Res.Aca.Rev.2016; 4(1): 77-90

Table.4 The Features of the Coronal Mass Ejections that have been used

\begin{tabular}{|c|c|c|c|c|c|}
\hline NO. & \multicolumn{2}{|c|}{$\begin{array}{c}\text { First C2 Appearance } \\
\text { Date Time }(\mathrm{UT})\end{array}$} & $\begin{array}{c}\text { Radius } \\
\boldsymbol{R}(\mathbf{K m})\end{array}$ & $\begin{array}{c}\text { Mass } \\
(\mathbf{K g})\end{array}$ & $\begin{array}{c}\text { Mass density } \\
\left(\mathbf{K g} / \mathbf{m}^{\mathbf{3}}\right)\end{array}$ \\
\hline 1 & $2011 / 03 / 07$ & $20: 00: 05$ & 2.43 & $1.7 \times 10^{13}$ & $8.4114 \times 10^{32}$ \\
\hline 2 & $2014 / 04 / 18$ & $13: 25: 51$ & 5.49 & $4.5 \times 10^{13}$ & $1.9307 \times 10^{32}$ \\
\hline
\end{tabular}

Table.5 Initial and Boundary Conditions of the Present Code for (coronal mass ejection (1))

\begin{tabular}{|c|c|c|}
\hline Quantity & Initial value & Boundary value \\
\hline Mass density, $(\rho)$ & $10^{-13}$ & 1.5 \\
\hline Particles velocity, $\left(\mathrm{u}_{\mathrm{x}}, \mathrm{v}_{\mathrm{y}}, \mathrm{w}_{\mathrm{z}}\right)$ & 0 & 10.00 \\
\hline Magnetic field, $\left(\mathrm{B}_{\mathrm{x}}, \mathrm{B}_{\mathrm{y}}, \mathrm{B}_{\mathrm{z}}\right)$ & $40 \times 10^{-4}$ & $10 \times 10^{-4}$ \\
\hline Pressure,$(\mathrm{p})$ & $10^{-7}$ & $10^{-8}$ \\
\hline
\end{tabular}

Table.6 Initial and Boundary Conditions of the Present Code for CME 2

\begin{tabular}{|c|c|c|}
\hline Quantity & Initial value & Boundary value \\
\hline Mass density, $(\rho)$ & $10^{-15}$ & 0.00 \\
\hline Particles velocity, $\left(\mathrm{u}_{\mathrm{x}}, \mathrm{v}_{\mathrm{y}}, \mathrm{w}_{\mathrm{z}}\right)$ & 1 & $2 \times 10^{-3}$ \\
\hline Magnetic field, $\left(\mathrm{B}_{\mathrm{x}}, \mathrm{B}_{\mathrm{y}}, \mathrm{B}_{\mathrm{z}}\right)$ & $10^{-6}$ & 2.00 \\
\hline Pressure, $(\mathrm{p})$ & $10^{-10}$ & $10^{-11}$ \\
\hline
\end{tabular}

Figure.1 Simulation of a Mass Density (kg. m-3) of CME1 Emerging from Sun into Interplanetary in Term of $\mathrm{R} \odot$ in Two Cases, when $\mathrm{Z}=5 \mathrm{R} \odot$ and $\mathrm{Z}=30 \mathrm{R} \odot$, using Three-

Dimension Ideal MHD Model
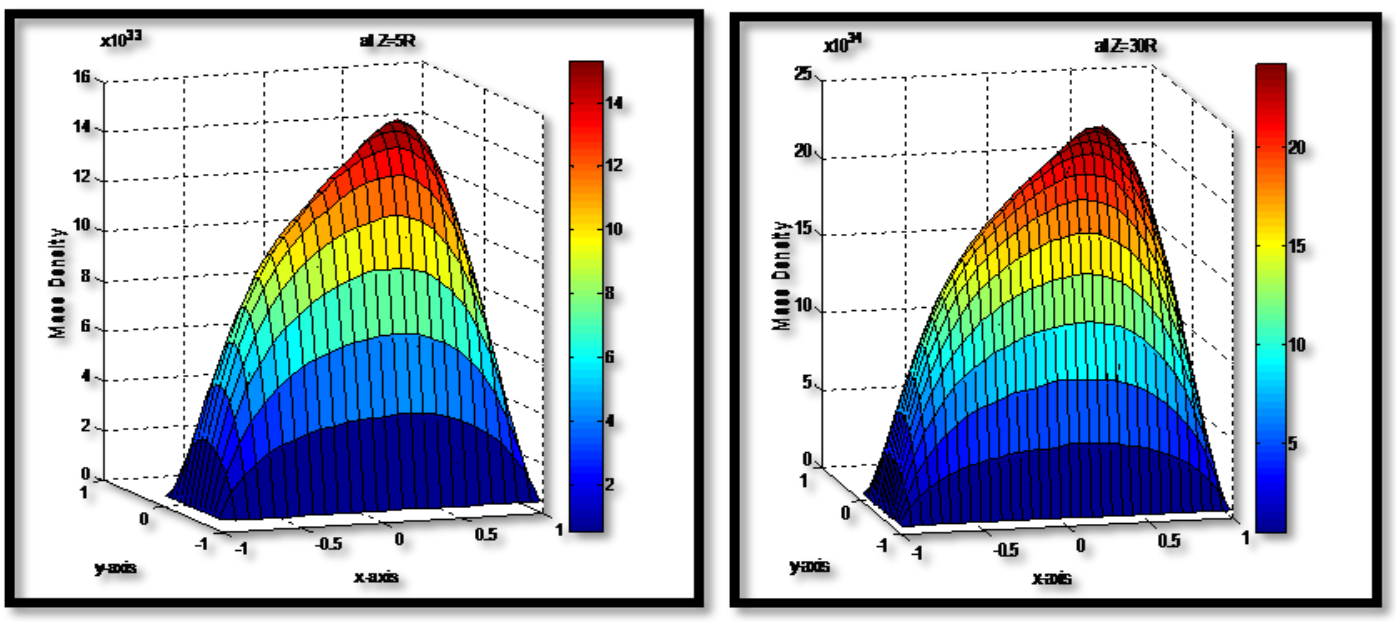
Int.J.Curr.Res.Aca.Rev.2016; 4(1): 77-90

Figure .2 Simulation of a Velocity (km.s-1) of CME1 Emerging from Sun into Interplanetary using Three-Dimension Ideal MHD Model
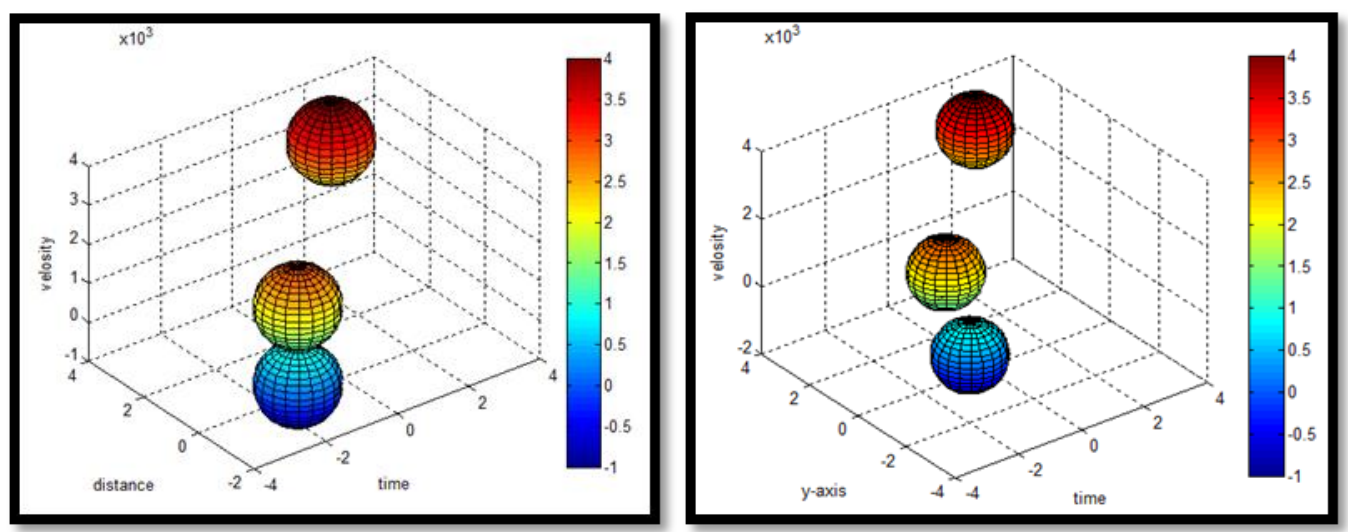

Figure.3 Simulation of a Mass Density (kg. m-3) of CME2 Emerging from Sun into Interplanetary in Term of $\mathrm{R} \odot$ in Two Cases, when $\mathrm{Z}=5 \mathrm{R} \odot$ and $\mathrm{Z}=30 \mathrm{R} \odot$, using ThreeDimension Ideal MHD Model
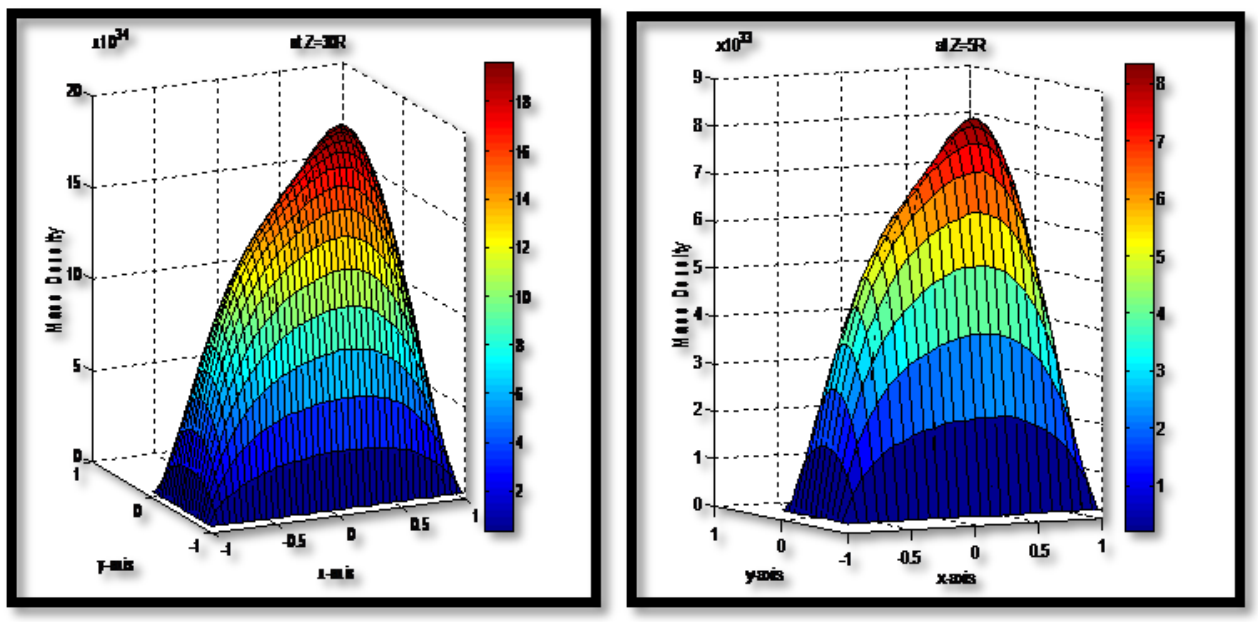

Table.7 Initial and Boundary Conditions of the Present Code for Jupiter

\begin{tabular}{|c|c|c|}
\hline Quantity & Initial value & Boundary value \\
\hline Mass density, $(\rho)$ & 0.01 & 0.09 \\
\hline Particles velocity, $\left(\mathrm{u}_{\mathrm{x}}, \mathrm{v}_{\mathrm{y}}, \mathrm{w}_{\mathrm{z}}\right)$ & 1.5 & 10.00 \\
\hline Magnetic field, $\left(\mathrm{B}_{\mathrm{x}}, \mathrm{B}_{\mathrm{y}}, \mathrm{B}_{\mathrm{z}}\right)$ & 0.1 & 0.8 \\
\hline Pressure,$(\mathrm{p})$ & 0.045 & 0.360 \\
\hline
\end{tabular}


Figure .4 Simulation of a Velocity (km.s-1) of CME2 Emerging from Sun into Interplanetary using Three-Dimension Ideal MHD Model, z=20
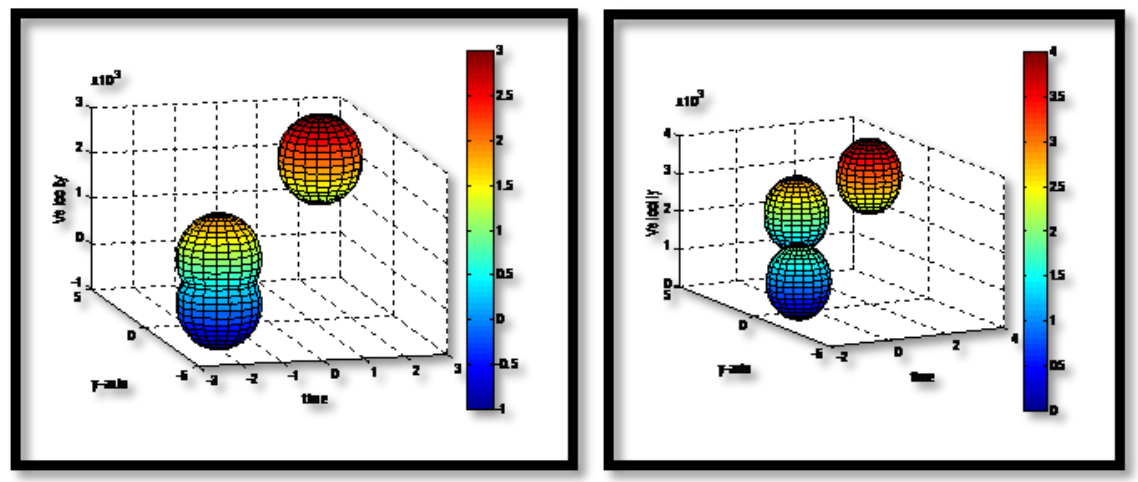

Figure.5 Simulation of the Magnetic Field in (nT) for CME1and CME2 that Emerging from Sun into Interplanetary by using Ideal MHD Model, $z=20$
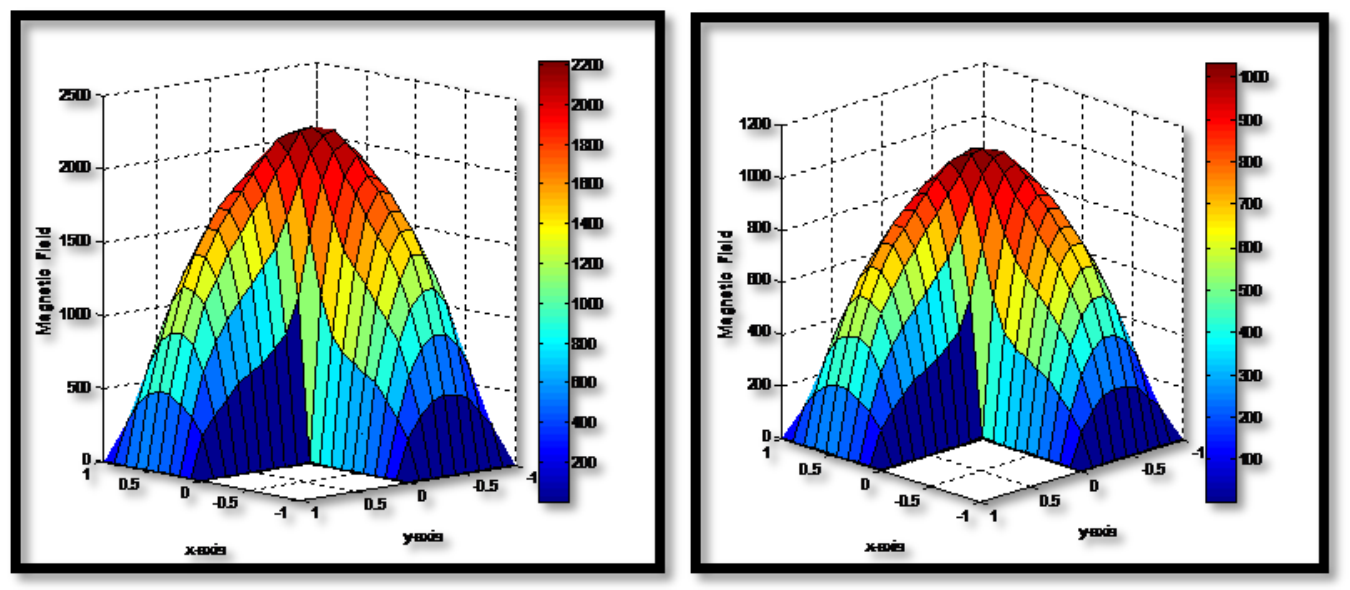

Figure.6 Simulation of the Magnetic Fields of Jupiter with B_Z component o of 0.75R_J equatorial plane. The Color Bar gives the Magnetic Field in (nT) Values. The Top Snapshots were taken $28.05 \mathrm{~h}$ after a Northward IMF of 0.105 nT Entered the Simulation Box. For this Simulation the V_X=300 km/s, $\mathrm{n}=8 \times \llbracket 10 \rrbracket^{\wedge} 4 \mathrm{~m}^{\wedge}(-3)$ and $\mathrm{T}=2 \times \llbracket 10 \rrbracket^{\wedge} 5 \mathrm{k}$

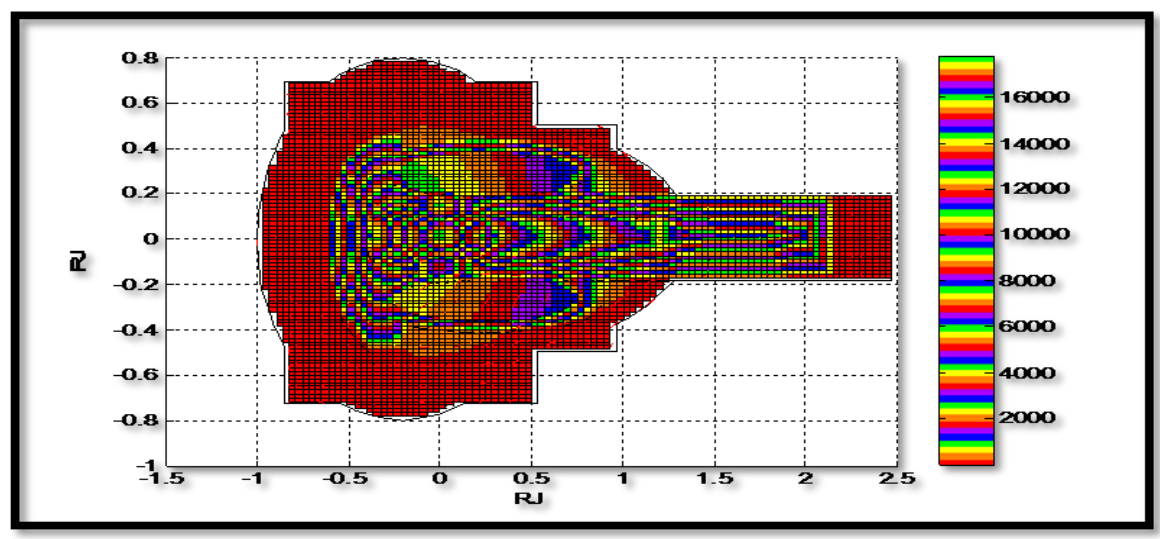


Figure.7 Simulation of the Interaction between the Magnetic field of CME1 (nT) and Jupiter Magnetic Field using Two-Dimension Semi-Relativistic MHD Model. The Color Bar Gives the Magnetic Field in (nT) Values

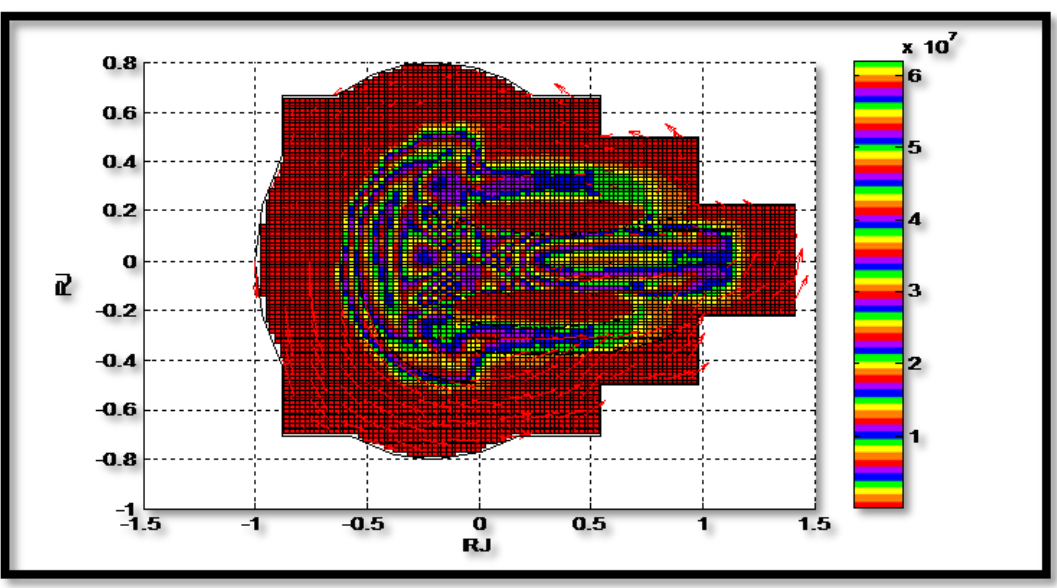

Figure.8 Simulation of the Interaction between the Magnetic field of CME2 (nT) and Jupiter Magnetic Field using Two-Dimension Semi-relativistic MHD Model. The Color Bar gives the Magnetic Field in (nT) Values

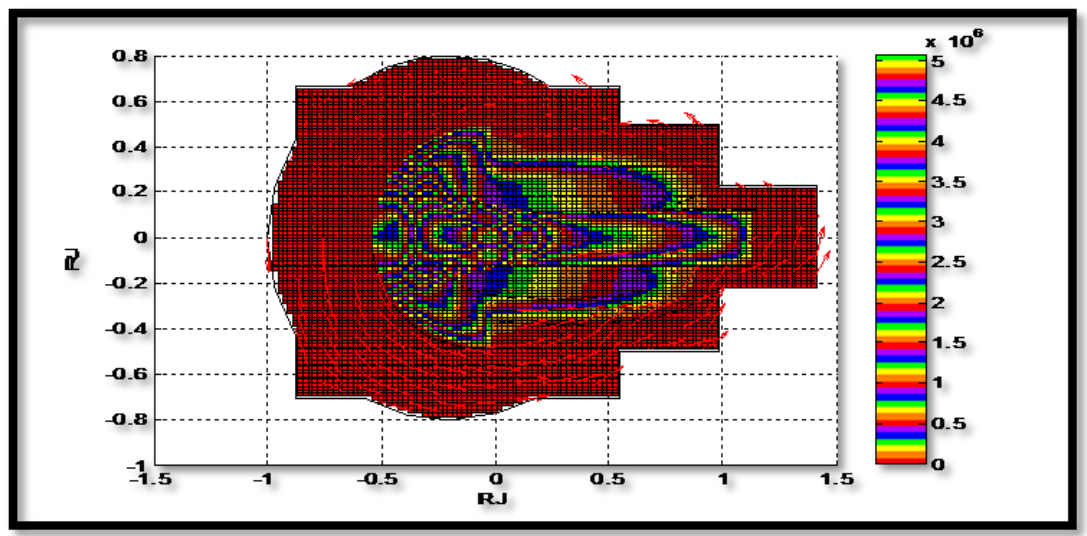

Figure.9 Simulation of Pressure Contours in the Dawn-dusk Meridian Plane as a Result of Interaction between CME1 and Jupiter using the Semi- Relativistic MHD Model. The Color Bar gives the Pressure (nPa) Values

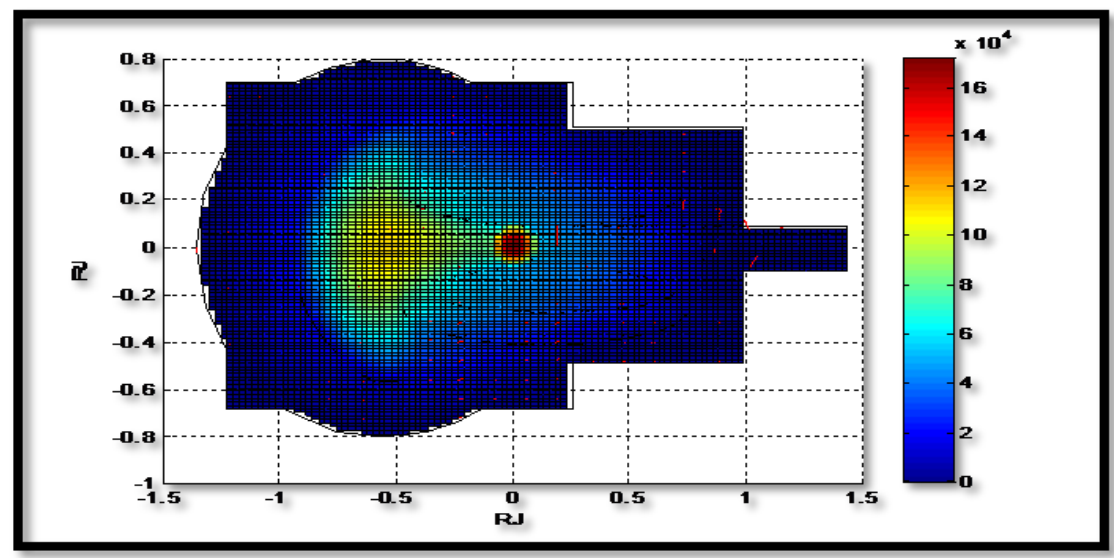


Figure.10 Simulation of Pressure Contours in the Dawn-dusk Meridian Plane as a Result of Interaction between CME2 and Jupiter using the Semi- relativistic MHD Model. The Color Bar gives the Pressure (nPa) Values

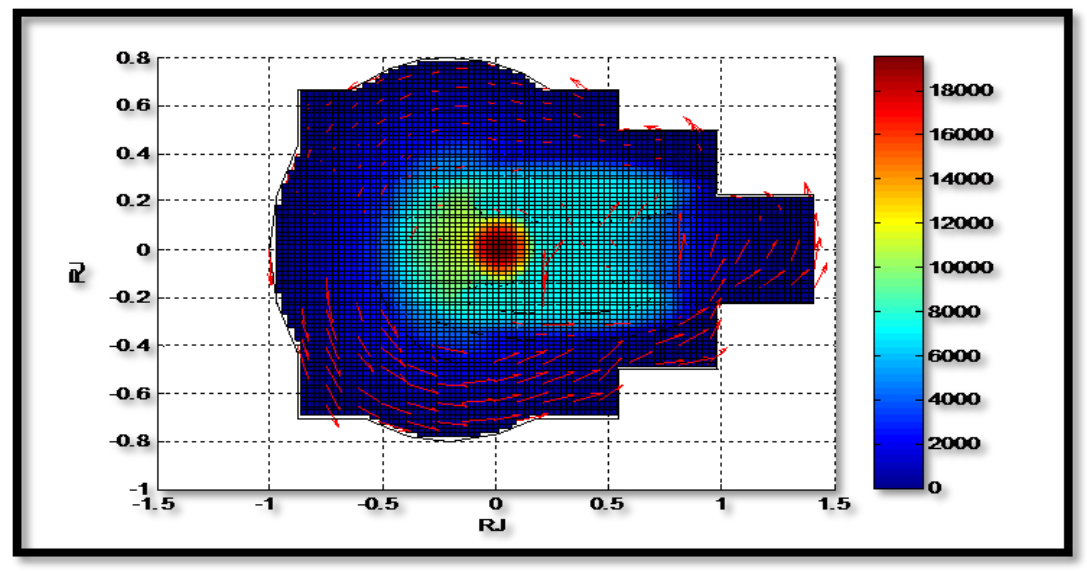

The north-south component of the magnetic field and the flow vectors and magnitude have been plotted in figure (6) by taking $28.05 \mathrm{~h}$ and $34.28 \mathrm{~h}$ after the northward turning of the IMF. The white line again shows $B_{Z}=0$. These waves started at about $18.7 \mathrm{~h}$ on the pre-noon magnetopause just like at Saturn. However at Jupiter the waves propagate tail ward along the dusk magnetopause rather than the dawn magnetopause. This occurs because the shear at Jupiter is primarily due to rotating Jovian flows rather than the solar wind and since the rotating flow goes counterclockwise toward dusk so do the waves. In the simulations reconnection occurred $80 R_{J}$ to $120 R_{J}$ down the tail with periods between $34 \mathrm{~h}$ and $100 \mathrm{~h}$. The onset of K-H waves occurs when flux tubes from the tail drift around to the dayside and increase the rotational velocity near the late morning magnetopause. Also figure (7) \& figure (8) represents the simulation of interactions of the magnetic field of CME1 \&CME2 respectively with the magnetic field of Jupiter using semirelativistic MHD model.

Figure (9) represents a thermal pressure in dawn-dusk meridian plane for the solar wind (CME1) dynamic pressures. The IMF was set to zero for these simulations. The pressures range from $0.045 \mathrm{nPa}$ to $0.36 \mathrm{nPa}$ and were selected by scaling the mean dynamic pressure at Jupiter's orbit, $0.092 \mathrm{nPa}$, by factors of two. Both the bow shock and magnetopause move toward Jupiter with increasing pressure. At noon the ratio of the bow shock distance to the magnetopause distance is between 1.31 and 1.24. It decreases with increasing pressure. Within the magnetosphere the equatorial plasma sheet is much thinner at dawn than dusk. This is reflected in an irregularly shaped magnetopause in the $X=0$ plane. However the dawn-dusk asymmetry and irregular shape of the magnetopause are much less evident in the bow shock. The dawn dusk asymmetry may actually reverse for small dynamic pressure.

Figure (10) represents the pressure in the YZ plane from a simulation for which the IMF is in the Y-direction $\left(B_{y}=0.42 n T\right)$ pointing toward dusk. The solar wind (CME2) dynamic pressure is $0.09 \mathrm{nPa}$. The corresponding fits to the $B_{I M F}=0$ bow shock and magnetopause positions are shown with solid and dashed lines respectively. The entire magnetosphere rotates about the sun-Jupiter line for $B_{y} \neq 0$. 
At high latitudes the boundaries are farther from Jupiter than for $B_{I M F}=0$ while nearer the equator they are closer to Jupiter. Reconnection can occur near the equator on the flanks of the magnetopause when the IMF points in the y-direction. This can change the shape of the obstacle. The addition of IMF $B_{y}$ does not change the standoff distance at the bow shock or the magnetopause. The standoff ratio remains 1.31 .

\section{Conclusion}

From the two applied methods which this work utilizes, the fixed path-length and the velocity dispersion analyses VDA, both are efficacious for these analyses. It should be noted events locate above $750 \mathrm{Km} / \mathrm{s}$, while most of the angles is $360^{\circ}$ (halo). The average heliocentric height of CME at first protons injection time with fixed path length method is 5.5729 solar radii, the corresponding height with VDA is 4 solar radii. In this study the profiles of protons intensities have clear correlations not to velocity but width of the CMEs of type (halo) is $360^{\circ}$. The mid-point leapfrog method has a higher (second) order of accuracy and it is suitable for this investigation, stable and more accurate from other approximations.

Generally, the results showed that:

a. When changing the coronal mass ejection type with different physical properties it has been noted that the results have been obtained from these CMEs are slightly changed in $\mathrm{B}$ and $\mathrm{V}$ results while the results of $\rho$ changed more rapidly.

b. When the spatial coordinate has been increased, it is seen that the part of the information about the Jupiter's magnetic field and CMEs interaction will be lost; this is due to the fact that increasing the mesh step will lead to improper solution MHD equation.

c. When increasing the time coordinates it has been noted that more details about the interaction between the CMEs and Jupiter's magnetic field can be obtained.

d. Finally, when studying the values of time iteration has been infer that.

- $\quad(\mathrm{dt}>0.1)$ there are no results i. e., no solution could be found.

- $\quad(\mathrm{dt}=0.1)$ the result is non-stable.

- $\quad(\mathrm{dt}<0.1)$ the results are stable and still constant. And also the increasing of mesh size will increase runtime drastically, so proper choice of mesh will give a best numerical results.

From the results of mass density it has been noticed that as the height increase, the peak decreases and the behavior become broader. This was explained due to the large force exerted from the solar activity. It has been noticed from the simulation that the resultant density of the magnetic field after the interaction between the CME magnetic field and Jupiter magnetic field is increase as the speed of the CME is increase. And a fast MHD shock with a convex surface which faces outward created because in this study the coronal mass ejection in speed more than Alfven speeds.

\section{References}

1. Encrenaz, Thérèse; Bibring, J.-P.; Blanc, M. 2003. The Solar System, Springer. ISBN 3-540-00241-3.

2. Kallenrode, May-Britt. 2004. Space Physics: An Introduction to Plasmas, Springer. ISBN 3-540-20617-5. 
3. Carroll, Bradley W.; Ostlie, Dale A. 1995. An Introduction to Modern Astrophysics, (revised 2nd ed.). Benjamin Cummings. ISBN 0-20154730-9. p. 409

4. Schrijver, Carolus J.; Zwaan, Cornelis. 2000. Solar and Stellar Magnetic Activity, Cambridge University Press. ISBN 0-521-58286-5.

5. Meyer-Vernet, Nicole., Basics of the Solar Wind, Cambridge University Press. ISBN 0-521-81420-0, (2007).

6. McComas, D. J., Elliott, H. A., Schwadron, N. A., Gosling, J. T., Skoug, R. M., \& Goldstein, B. E., The threedimensional solar wind around solar maximum, Geophys. Res. Lett., 30(10):100000-1, (2003).

7. Brueckner, G. E., Howard R.A., Koomen M. J., Korendyke C. M., Michels, D.J., Moses, J.D., Socker, D.G., Dere, K.P., Lamy, P.L., Liebaria, A., Bout M.V., Schwenn, R., Simnett, G. M., Bedford, D.K., Eyles, C.J., Solar Physics 162:357-402(1995).

8. Toth, G.,Holst,B.V., Sokolove, I.V., Zeeuw, D.L., 2012. Adaptive numerical algorithms in space weather modeling, Journal of computational physics 23: 870-903

9. Delamere, P. A., and Bagenal, F.2010. Solar wind interaction with Jupiter's magnetosphere, J. Geophys. Res. 115, A10201. doi: 10.1029/2010JA015347.

10. Thomas Wiegelmann. 2012. Space Plasma Physics.

11. Lugaz, N., Manchester IV, W.B and Gombosi, T.I. 2005. The Evolution Of Coronal Mass Ejection Density
Structures, Center for Space

Environment Modeling, University of Michigan, Ann Arbor, MI Received 2004 December 3; accepted 2005 March 2. The Astrophysical Journal, 627:10191030, 2005 July 10. The American Astronomical Society. All rights reserved. Printed in U.S.A..

12. Wegmann, R., 1995, MHD Model for the Effect of Interplanetary Shocks of the Plasma Tail of Comet, Astro. Astrophy., 294: 601-616, and Rauer, H., Wegmann, R., Schmidt, H. U., Jockers, K., 1995, 3-D MHD Simulation of the Effect of Comoving Discontinuity in the Solar Wind on Cometary Plasma Tails, Astro. Astrophys. 295: 529-550.

13. Meng, X., Tóth, G., Sokolov, I.V. and Gombosi, T.I. 2012 Classical and Semi relativistic Magneto hydrodynamics with Anisotropic Ion Pressure, Journal of Computational Physics, doi: 10.1016/ j.jcp.2011.12.042.

14. Boris, J. P., A physically Motivated Solution of the Alfven Problem, Tech. Report NRL Memorandum Report 2167 (Naval Research Laboratory, Washington, DC, 1970).

15. Klaus A. Hoffmann and Steven $T$. Chiang, 2000, Computational Fluid Dynamics, Fourth edition, vol.1, ch.6.

16. Seidelmann, P. Kenneth; Archinal, Brent A.; A'Hearn, Michael F. et al. 2007. Report of the IAU/IAG Working Group on cartographic coordinates and rotational elements: 2006, Celestial Mechanics and Dynamical Astronomy 98 (3): 155-180.

\section{How to cite this article:}

Wafaa A. Zaki and Zainab A. Ali. 2016. Studying the Interaction between the CME Magnetic Field and Jupiter's Magnetic Field by Simulation. Int.J.Curr.Res.Aca.Rev. 4(1): 77-90. doi: http://dx.doi.org/10.20546/ijcrar.2016.401.008 